\title{
QOS MANAGEMENT IN REAL-TIME SPATIAL BIG DATA USING FEEDBACK CONTROL SCHEDULING
}

\author{
S. Hamdi ${ }^{\mathrm{a}, *}$, E. Bouazizi ${ }^{\mathrm{b}}$, S. Faiz \\ ${ }^{a}$ Tunisia Polytechnic School, BP 2078 La Marsa, University of Carthage, Tunisia - hamdisana@ gmail.com \\ ${ }^{\mathrm{b}}$ MIRACL Laboratory, Higher Institute of Computer Science and Multimedia, Sfax University, Tunisia - emna.bouazizi@gmail.com \\ ${ }^{\mathrm{c}}$ LTSIRS Laboratory, BP 37 Le Belvedere 1002, Tunis, Tunisia - sami.faiz@ insat.rnu.tn
}

Commission II, WG II/4

KEY WORDS: Geographic Information System, Real-Time Spatial Big Data, Heterogeneous Real-Time Geospatial Data, Transaction, Feedback Control Scheduling, Quality of Service.

\begin{abstract}
:
Geographic Information System (GIS) is a computer system designed to capture, store, manipulate, analyze, manage, and present all types of spatial data. Spatial data, whether captured through remote sensors or large scale simulations has always been big and heterogenous. The issue of real-time and heterogeneity have been extremely important for taking effective decision. Thus, heterogeneous real-time spatial data management has become a very active research domain. Existing research has principally focused on querying of real-time spatial data and their updates. But the unpredictability of access to data maintain the behavior of the real-time GIS unstable. In this paper, we propose the use of the real-time Spatial Big Data and we define a new architecture called FCSA-RTSBD (Feedback Control Scheduling Architecture for Real-Time Spatial Big Data). The main objectives of this architecture are the following: take in account the heterogeneity of data, guarantee the data freshness, enhance the deadline miss ratio even in the presence of conflicts and unpredictable workloads and finally satisfy the requirements of users by the improving of the quality of service (QoS).
\end{abstract}

\section{INTRODUCTION}

In recent years, spatial applications have become more and more important in both scientific research and industry. The challenge of these applications has always been how to represent and understand the fast-paced, constantly changing world, given increasingly real-time data including readings from real world (Xu, 2013).

Traditional static GIS pays more attention to representing historic data and temporal GIS only treats time as a occasional but not critical factor and can't support the explicit change representation. In this context, real-time GIS is put forward as one potential development in the future of GIS (Goodchild, 2012). It gives users the ability to connect to real-time data streams, perform continuous processing and analysis of those data streams, and send relevant information to users or other systems.

Since information technology is innovating on the way we live, there is tremendous amount of heterogeneous real-time spatial data generated everyday. As a result, the growth of the data volume seems to outspeed the advance of our computing infrastructure. Conventional data processing technologies, such as database and data warehouse, are becoming inadequate to the amount of data we want to deal with. This new challenge is known as realtime spatial Big Data (Van, 2014).

In the systems that manage the real-time spatial Big Data, transactions arrive at varying frequencies. As the frequency increases dramatically, the balance of these systems is jeopardized. During the periods of overload, real-time spatial Big Data will potentially run out of resources and real-time transactions will then miss their deadline in greater numbers. In this paper, we propose a new approach called FCSA-RTSBD (Feedback Control Scheduling Architecture for Real-Time Spatial Big Data) to manage the QoS in the real-time spatial Big Data. In section 2, we discuss the

${ }^{*}$ Corresponding author related works. Then, in section 3, we present the system model that we consider. In section 4, we propose an approach of QoS management in real-time spatial Big Data. Finally, we present our conclusions in section 5 .

\section{RELATED WORKS}

Researching on the real-time spatial analysis is a hot topic in Spatial Big Data. Nowadays, spatial characteristics can be used in real-time Spatial Big Data analysis, providing the necessary information and knowledge to optimize processes and to solve problems in real-time GIS.

In (Boyd, 1987), once the positions and other spatial properties of objects and events are clear, real-time spatial analysis can be performed, and based on this analysis, decisions can be made. This process is described by a continuous loop of sensing, analyzing, predicting, and actuating in a Smart City called OODA (Observe-Orient-Decide-Act) loop where a real-time spatial analysis of event streams is realized by integrating spatial algorithms in a SOA-EDA (Service Oriented Architecture-Event Drive Architecture) configuration (Zeimpekis, 2006).

In (Marz, 2014), the authors proposed an architecture called Lambda architecture. It is a data-processing architecture designed to handle massive quantities of data by taking advantage of both batch and stream-processing methods. This approach to architecture attempts to balance latency, throughput, and fault-tolerance.

In (Martnez, 2014), Martnez and al, based on the foundations of the Lambda architecture, proposed an other architecture called SOLID (Service-On-Line-Index-Data architecture): an architecture for real-time management of big semantic data which separates the complexities of big semantic data storage and indexing from real-time data acquisition and consumption. In this architecture, historical data and real-time data are both stored. It ensures efficient volume management and high processing velocity. 
In fact, SOLID is a good architecture for the real-time management of big semantic data. But it does not take into account the heterogeneity of real-time geospatial data. Thus, our work is to introduce a specific management of the heterogeneous real-time geospatial data based on techniques of scheduling with feedback control called FCSA-RTSBD.

\section{SYSTEM OVERVIEW}

In this section, we give an overview of real-time spatial Big Data, heterogeneous real-time geospatial data model and transaction model.

\subsection{Real-time spatial Big Data}

GIS are distinguished from other information systems in several aspects and we can note the following points (Cova, 2014):

- The complexity of spatial data structures (as opposed to traditional data types of the DBMS).

- An important amount of data (up to Terra bytes in the case of large systems containing raster data).

- The coexistence of many formats or geographical models.

The growth of spatial data (specially real-time spatial data) has been explosive thanks to cost-effective and ubiquitous positioning technologies, and the generation of data from multiple sources in multi-forms. Nowadays, current GIS (real-time GIS) manage huge real-time spatial data. A widely accepted meaning says that Big Data is "when the size of the data itself becomes part of the problem" (Van, 2014). Thus, we propose the use of real-time spatial Big Data to process such large amount of heterogeneous data (Loukides, 2012) which is characterized by:

- High-volume: Big Data represent large amounts of data. It is generally said that $90 \%$ of the data available today were created in the last two years (Lodha, 2014).

- High-velocity: Data are generated, captured and shared with an important speed. The delays of actualization and analysis of the spatial data are short and they are treated in real-time.

- High-variety (or heterogeneity): Analyzed data are not necessarily structured. It can be also unstructured data.

\subsection{Heterogeneous real-time geospatial data model}

As spatial data is collected, it is stored as spatial entities in the computer as points, lines, and areas which is represented in layers in the spatial model of GIS. These layers when combine create a map. When these features of points, lines and areas are needed to be stored they use one of two models: Raster and Vector. Raster model stores continuous geographic data in cells within a grid of a layer. Vector model stores discrete geographic data like road networks, rivers as points.

The term real-time refers to the capability of management, visualization and analysis of graphical and attribute information as soon as an event occurs (Nadi, 2003) and a real-time spatial data is a representation of the entities of the real world composed of an identity, descriptive properties and spatial properties. While the identity describes a stationary semantics of the entity, the descriptive and spatial properties can vary in the time and constitute the dynamic part of the entity.

Real-time spatial applications have a great importance. Examples of these applications are:
- Emergency management (Cova, 2014).

- Hazards/disaster management (Coleman, 1996).

- Public health including real-time outbreak and disease surveillance (RODS) (Tsui2003a).

- Transportation including management of highway incidents (Lepofsky, 1995), online feet management and dynamic routing of the feet in case of congestion using real-time traffic information (Schafer, 2002).

Stored data in these applications are from heterogeneous sources and are maintained under heterogeneous formats and structures. These data can be divided into two types: the structured data and unstructured data:

- Structured data: Structured data can be processed automatically by machines. As mentioned in (Bishr1998a) (Stuckenschmidt, 2005) that illustrate structured data heterogeneity through three levels, i.e., semantic schematic and syntactic.

- Syntactic heterogeneity refers to data format differences. Some organizations are motivated to make their data accessible through Open Geospatial Consortium (OGC) formats that provide syntactically unified formats and services such as Geography Markup Language (GML) (Cox, 2003) and Web Feature Service (WFS) (Zhang, 2005).

- Schematic heterogeneity refers to data model differences. Each spatial database schema reflects an abstracted view of data. Different hierarchical and classification structures are used to refer to identical or similar objects. Hence, schematic heterogeneity can be classified into three main schemes:

* Entity versus Entity, i.e., the same entity can exist in two different databases with different name or structure;

* Attribute versus Attribute, i.e., an attribute related to a class in one database can exist in another class related to another database;

* Entity versus Attribute, i.e., a class in one database can be designed as an attribute in another database.

- Semantic heterogeneity can be divided into two types: the naming heterogeneity and the cognitive heterogeneity.

* The naming heterogeneity arises when the same data objects are named in a different manner or when different semantic data objects are named identically.

* The cognitive heterogeneity refers to the different domains assigned to each organization.

- Unstructured data: Unstructured data are those in which the information is within simple text that no common pattern can be used to process and come from different sources and have a different format as text, pictures, multimedia content or numeric traces, etc.

In our work, heterogeneous real-time geospatial data challenges are essentially data integration and cleaning, data reduction and data indexing and query: 
1. Real-time geospatial data integration and cleaning: Since the data is heterogeneous, it is not enough merely to record it. Data are required to be expressed in forms that are computer understandable and then resolvable. In real-time spatial Big Data, we are talking about two levels of heterogeneity (unstructured data - structured data) and (Syntactic heterogeneity - Schematic heterogeneity - Semantic heterogeneity), we have to think first of integrating unstructured data and structured data; it is important to be able to analyze unstructured data as well as integrate unstructured data with structured data, and as a second step we have to handle the heterogeneous structured data.

2. Real-time geospatial data reduction: Data reduction is the reduction of multitudinous amounts of data by the transformation of numerical or alphabetical digital information derived empirically or experimentally into a corrected, ordered, and simplified form. Because real-time geospatial data is complex, we will often find that the data objects have high dimensionality; each data object is annotated with a large number of values. The types of values that are shared among all the different data objects are usually referred to as parameters. It is very difficult to make much sense of high dimensional data. It is always best to develop a filtering mechanism that expunges useless parameters. A useless parameter will often have one of these two properties.

- Redundancy: If a parameter correlates perfectly with some other parameter, you know that you can safely drop one of the two parameters.

- Randomness: If a parameter is totally random, then it cannot tell you anything meaningful about the data object and you can drop the parameter.

3. Real-time geospatial data query and indexing: The complex physical organization of real-time geospatial data storing is considered. The most important requirements for these data structures are the ability of providing fast access to the large volumes of data.

\subsection{Transaction model}

Spatial real-time transactions can be classified into two classes: update transactions and user transactions (Martnez, 2014).

- Update transactions: update the values of real-time spatial data in order to reflect the state of real world.

- User transactions (continuous requests): user requests, arrive aperiodically and may read real-time data and non realtime data.This type of transaction is executed several times continuously as required by the user.

These transactions are characterized by $\mathrm{ACID}^{1}$ properties as follow:

- Atomicity: All transactions are executed successfully, or not at all (all-or-nothing). If that fails, the system must cancel all modifications that have been made by the transaction.

- Consistency: A transaction must pass the base from one consistent state to another state coherent. If unsuccessful, the initial state must be restored.

\footnotetext{
${ }^{1}$ (Atomicity, Consistency, Isolation, Durability)
}

- Isolation: Transactions are called isolated from each other, i. e that their effects on the database become visible to other transactions only when they perform the commit operation (COMMIT).

- Durability: When a transaction is committed, its effects on the database become permanent. The system must ensure that its modifications (updates) will be retained.

\section{QOS MANAGEMENT IN REAL-TIME SPATIAL BIG DATA}

In this section, we propose a definition of QoS in the real-time Spatial Big Data and we define a new architecture called FCSARTSBD as a new QoS management approach in real-time GIS.

\subsection{QoS definition}

The QoS can be seen as a metric that permits to measure the overall system performance [22]. Indeed QoS is a collective measure of the service level provided to the customer. It is characterized by different performance criteria.

\begin{tabular}{|l|l|}
\hline Criteria & Definition \\
\hline Lineage & $\begin{array}{l}\text { Description of the history of the } \\
\text { data }\end{array}$ \\
Attribute accuracy & $\begin{array}{l}\text { Evaluation of the gaps between the } \\
\text { nominal positions and the positions } \\
\text { of the data collected in the data } \\
\text { base } \\
\text { Difference between the values of } \\
\text { the attributes and the values of their } \\
\text { counterparts in the nominal land. } \\
\text { Appropriateness in the data repre- } \\
\text { sentation for user. } \\
\text { Internal consistency of the data ac- } \\
\text { cording to the rules of modeling. }\end{array}$ \\
\hline
\end{tabular}

Table 1: Evaluation criteria of data quality.

In static GIS, the QoS is defined by criteria stored in meta-data identified by ISO standards as shown in Table 1. These criteria of quality are defined for static data used by traditional applications and were designed for geographic data producers.

These criteria do no take into account the qualification of realtime. Besides, there is not a detailed definition for the QoS in real-time GIS. Thus, we propose to inspire the definition of the QoS in real-time spatial Big Data from of the definition of the QoS in RTDBSs ${ }^{2}$ and RTDWHSs ${ }^{3}$. In these systems, the notion of QoS is defined through two concepts the quality of data (QoD: precision and freshness) and the quality of transaction (QoT) which are enhanced by alleviating the risk of transaction miss deadline (Ramamritham, 2004)(Leng, 2011) but it doesn't treat spatial data.

As a result, we propose the definition of QoS in real-time spatial Big data through two concepts:

- Quality of data (QoD): Evaluation criteria of QoD are the same for a static spatial data but we add new criteria to verify the precision and and the freshness of the real-time spatial data.

- Quality of transaction (QoT): Estimated by the percentage of the transactions that finishes their execution without missing their deadlines.

\footnotetext{
${ }^{2}$ Real-Time DataBase Systems

${ }^{3}$ Real-Time Data WareHouses Systems
} 


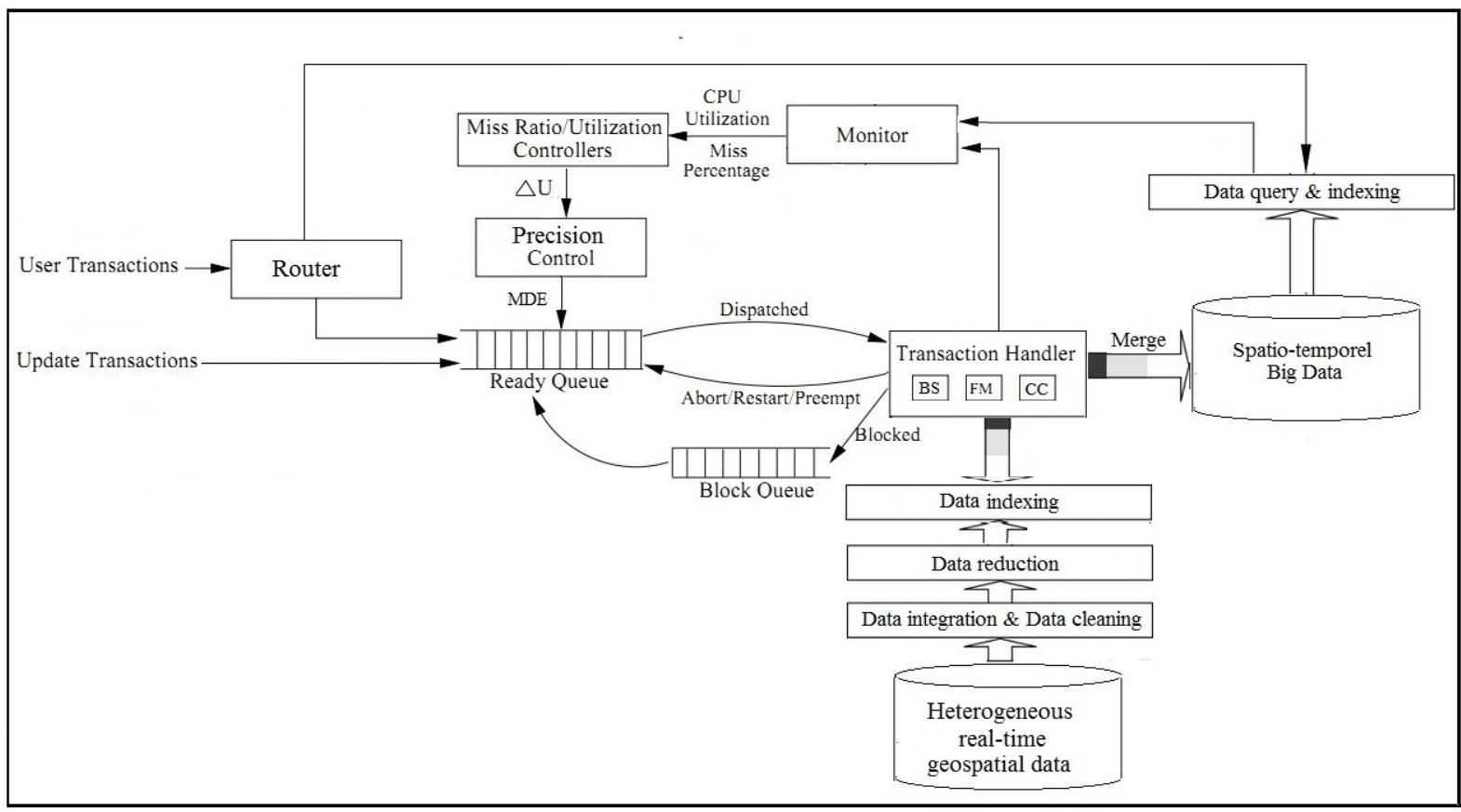

Figure 1: A Feedback Control Scheduling Architecture for Real-Time Spatial Big Data: FCSA-RTSBD.

\subsection{A Feedback Control Scheduling Architecture for Real- Time Spatial Big Data: FCSA-RTSBD}

In this section, we propose the design of FCSA-RTSBD that can provide data services with QoS guarantees. FCSA-RTSBD exploits a feedback control loop that we will explain. As shown in Figure. 1, the architecture consists of a router, a precision control, a handler and a monitor. Admitted transactions are placed in the ready queue. The transaction handler manages the execution of the transactions. The system performance statistics are collected periodically by the monitor. Below we briefly describe each component.

- Router: forwards user transaction according to the type of data reached by this transaction.

- Precision controller: rejects update transactions when the data update is sufficiently representative of the real world considering the value of $\mathrm{MDE}^{4}$ : it determines whether the transaction wishes to update a real-time data can be discarded $(\mathrm{DE}<\mathrm{MDE})$ or not $(\mathrm{DE}>\mathrm{MDE})$ with DE (Data Error) which refers to how the current state of the targeted environment may differ from the measured data (Ramamritham, 1993). The data error on a data version $d$ is defined by:

$$
D E(d)=100 \times\left|\frac{\text { currentvalue }(d)-\text { updatevalue }(d)}{\text { currentvalue }(d)}\right|
$$

current value $(d)$ denote the value of $d$ before update and update value $(d)$ denote the value of $d$ after update. Further, the precision controller decreases the response time of user transactions with access to fresh data by discarding any unnecessary freshness.

- Transaction handler: allows the execution of transactions ordered in ready queue, through additional modules such as:

\footnotetext{
${ }^{4}$ Maximum Data Error
}

- Freshness Manager (FM): checks the freshness of data.

- Concurrency Controller (CC): In computer systems the performance can be increased by allowing concurrent execution of operations. The activity of coordinating concurrent access of transactions to shared data is called concurrency control. Concurrency control algorithms should ensure consistency of the realtime spatial Big Data. Concurrency control protocols resolve data access conflicts in a manner that induces a serialization order among the conflicting transactions. The problem of concurrency control in traditional Big Data has been intensively studied and presented in literature (Dai, 2009). In FCSA-RTSBD, we must take into account spatial and timing constraints of transactions.

- Basic Scheduler (BS): Spatial real-time transactions should be scheduled considering both data consistency and timing constraints in such a way that they can be completed before their corresponding deadlines expire.

- Spatio-temporel Big Data: (Historical Big Data) it stores raw data. It can never be altered or deleted, only insertions are allowed.

- Merge: integrates the updated spatial data into the spatiotemporel Big Data then deletes its old version found in realtime spatial Big Data.

- Monitor: allows the measurement of system performance by monitoring the execution of transactions. It measures the system performance by inspecting an execution of transactions (number of transactions completed, abandoned, who missed deadline...). The measured values are part of the feedback control loop that helps to stabilize the system.

- Utilization controller: used to adjust the value of MDE.

FCSA-RTSBD exploits the foundations of the architecture of Aurora (Daniel2003) (see the Aurora architecture in Figure. 2). Aurora is a data-flow system that process incoming stream according 


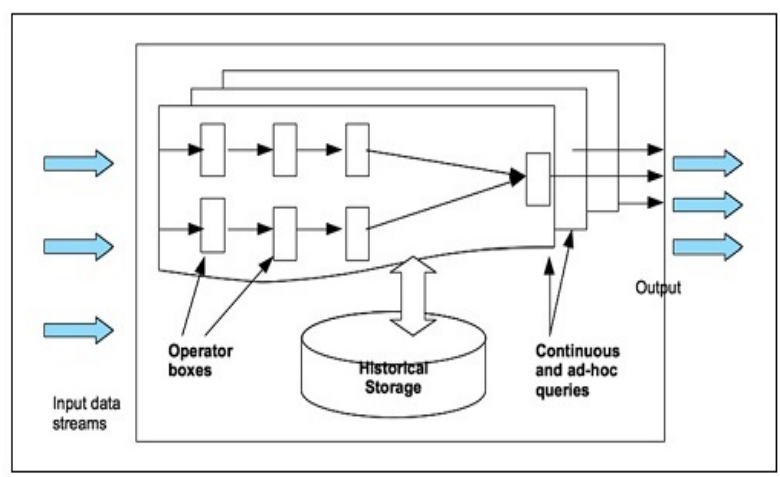

Figure 2: Aurora architecture (Daniel2003).

to the requirements of the applications. It includes a set of operators for satisfying the stream processing requirements. Each operator consumes data input, performs operations, and produces results in a continuous manner. Among these operators we can note: windowed operators, filter operator, etc. Aurora can process continual queries in real-time processing according to QoS specifications.

Thus, FCSA-RTSBD allows to limit the deadline miss ratio and to support freshness for the data accessed by timely transactions (even in the presence of unpredictable workloads). So the data used by transactions are fresh. FCSA-RTSBD permits also to guarantee the QoD and the QoT, and therefore it enhances the QoS. FCSA-RTSBD can perform very well in this context of changing user requirements due to the adjustability.

\section{CONCLUSION}

In this paper, we proposed a new approach called FCSA-RTSBD to maintain the behaviour of the real-time spatial Big Data in a stable state and satisfy the requirements of users. We used a precision control to control the incoming transactions according to CPU utilization of the system.

The advantage of our approach is to allow users to express their real needs for their queries and to deal with the transient overload to better manage the real-time spatial Big Data performance.

As follow, we plan to make simulations and to extend this work in several different ways. We will consider other aspects to study different components of the feedback control scheduling architecture for the QoS management in real-time spatial Big Data.

\section{REFERENCES}

Bishr, Y., 1998a. "Overcoming the semantic and other barriers to gis interoperability" International Journal of Geographical Information Science.

Boyd, J., 1987. ”A Discourse on Winning and Losing”.

Coleman, J., Sullivan, A., 1996 . "A real-time computer application for the prediction of fire spread across the Australian landscape", Simulation.

Cova, T., 2014. "GIS in emergency management", Geographical Information Systems: Principles, Techniques, Applications.

Cox, S., Daisey, P., Lake, R., Portele, C.,Whiteside, A., 2003. "Opengis geography markup language (gml) implementation specification, version $3.0 "$
Dai, J., Lu, C. T., Lai, L. F. 2009. ”A concurrency control protocol for continuously monitoring moving objects. In Mobile Data Management: Systems, Services and Middleware", Tenth International Conference on (pp. 132-141).

Daniel, A., Don, C., Ugur, C., Mitch, C., Christian, C., Sangdon, L., Michael, S., Nesime, T. , Stan, Z., 2003a. "Aurora: a new model and architecture for data stream management", $V L D B$ Journal.

Goodchild, M., 2012. "Looking Forward: Five Thoughts on the Future of GIS", Essays on Geography and GIS, vol 4, 26-29.

Leng, F., Bao, Y., Yu, G., Shi, J. and Cai, X., 2011. "Requirementbased Query and Update Scheduling in Real-time Data Warehouses", $12^{\text {th }}$ international conference on Web-age information management (WAIM).

Lepofsky, M., Abkowitz, M., Cheng, P., 1995. "Transportation hazard analysis in an integrated GIS environment", BeroggiGEGandWallace W A (eds) Computer Supported Risk Management. Amsterdam, Kluwer.

Lodha, R., Jain, H., Kurup, L., 2014. ”Big Data Challenges: Data Analysis Perspective".

Loukides, M., 2012.'Data Science and Data Tools”, Big Data Now, chapter 1. OReilly.

Martnez-Prieto, M. A., Cuesta, C. E., Arias, M., Fernndez, J. D., 2014. "The Solid architecture for real-time management of big semantic data", Future Generation Computer Systems.

Marz, N., Warren, J., 2014. "Big Data: Principles and best practices of scalable realtime data systems"

Nadi, S., Delavar, M. R., 2003. "Spatio-Temporal Modeling of Dynamic Phenomena in GIS", ScanGIS, 215-225.

Ramamritham, K.,1993. "Real-Time Databases", Distributed and Parallel Databases, vol. 1, n . 2, 199-226.

Ramamritham, K., Son, S., Dipippo, L., 2004. "Real-Time Databases and Data Services", Real-Time Systems , vol. 28, 179-215, Kluwer Academic Publisher.

Schafer, R., Thiessenhusen, K., Wagner, P. , 2002.”A trafic information system by means of real-time floating-car data", TS World Congress, Chicago, Illinois.

Stuckenschmidt, H., Harmelen, F., 2005. "Sharing on the Semantic Web"', Springer Berlin Heidelberg.

Tsui, F., Espino, J., Dato, V., Gesteland, P., Hutman, J., Wagner, M., 2003a.'Technical description of RODS: A real-time public health surveillance system", Journal of the American Medical Informatics Association .

Van der Zee, E., Scholten, H., 2014. "Spatial Dimensions of Big Data: Application of Geographical Concepts and Spatial Technology to the Internet of Things", Big Data and Internet of Things. A Roadmap for Smart Environments.

Wei, Y., Son, S.H., Stankovic, J.A., and Kang, K.-D., 2003. ”QoS Management in Replicated Real Time Databases", IEEE RTSS, 86-97. IEEE Computer Society.

Xu, W., Zhu, Q., Zhang, Y., Ding, Y., Hu, M., 2013. "RealTime GIS and its Application in Indoor Fire Disaster", ISPRSInternational Archives of the Photogrammetry, Remote Sensing and Spatial Information Sciences,121-127. 
Zhang, C., Li, W., 2005. "The roles of web feature and web map services in real time geospatial data sharing for time-critical applications", Cartography and Geographic Information Science, 269-283.

Zeimpekis, V., Kourouthanassis, P., Giaglis, G.M., 2006. ’Telecommunication Systems and Technologies”, vol. I. UNESCO-EOLSS. 